\title{
A Cu(II)-based Coordination Polymer: Treatment Activity on Tuberculosis by Inhibiting the Survival of the Mycobacterium in Alveolar Macrophages
}

\author{
Lei Zhao, Zhan-Ying Li, Tao Wang, Ying Qi, Pei Wang, and Li-Li Diao* \\ Division of Pulmonary Tuberculosis, the North Courtyard of Affiliated Hospital of Heibei University, Baoding, Heibei, CHINA
}

\begin{abstract}
Via utilizing the mixed-ligand method, a novel coordination polymer (CP) containing $\mathrm{Cu}$ (II) has been prepared by self-assembly at the ambient temperature, and its chemical formula is $\left\{\left[\mathrm{Cu}(\mathrm{BINDI})_{0.5}\right.\right.$ (bpe) $\left.\cdot 3 \mathrm{H}_{2} \mathrm{O}\right\}_{n}\left(1\right.$, bpe $=1,2$-bis(4-pyridyl)ethylene and $\mathrm{H}_{4} \mathrm{BINDI}=N, N^{\prime}$-bis(5-isophthalic acid $)$ naphthalenediimide). For the treatment of the tuberculosis, its biological function was evaluated as well. The CFU assay was performed to determine the bacterial numbers of the Mycobacterium in alveolar macrophages. In addition to this, the rop $A$ gene of the Mycobacterium in alveolar macrophages was also detected through the real time RT-PCR method. Only the oxygen atoms on the metal complex are identified to be able to interact with the probe protein by molecular docking simulation.
\end{abstract}

Key words: coordination polymer, tuberculosis, Mycobacterium, molecular docking

\section{Introduction}

Mycobacterium tuberculosis (MTB) is dominated by bacterial cell immunity in humans. Macrophages are the main host cells and effector cells of MTB. Their activity and function play a very important role in the MTB immune response and the outcome of the infection ${ }^{1)}$. Usually, the Mycobacterium could survival in the alveolar macrophages, it could multiply the nutrients in the cells, which is used for the proliferation of itself. When the concentration of the Mycobacterium reaches to a certain level, it will damage the alveolar macrophages and spread to other sites and cause the infection ${ }^{2,3)}$. Thus, in this present study, our purpose is to investigate novel candidates for the $\mathrm{Myco}^{-}$ bacterium tuberculosis treatment by targeting the Mycobacterium.

Recently, the structure along with design of the metal supramolecular structures on the basis of crystal engineering is a hot topic coordination chemistry and supramolecular chemistry fields ${ }^{4-6)}$. The reason why people are more and more interested in the above field is not only their worthy unit structures, but also their latent applications in biochemistry, catalysis and luminescence, especially in the field of modern pharmaceutical chemistry ${ }^{7-9)}$. Among a series of the prepared compounds, functional complexes have attracted much attention because of their latent medicinal value. Therefore, the selection of biocompatible, ef- ficient and safe ligands has become a key factor in the clinical applications, drug therapy and structural design field. The heterocyclic ligands containing nitrogen and the polydentate ligands (such as the polycarboxylic acids) have been extensively utilized in the reasonable design as well as the controlled synthesis of these multifunctional complexes $^{10-13)}$. In the years, $N$-heterocyclic carboxylic acid ligands have received wide attention of biologists and chemists due to their functional performances and rich coordination patterns, along with the hydrogen-bonding receptors and donors under the conditions of solution ${ }^{14-16)}$. In this current research, via utilizing the mixed-ligand method, a novel coordination polymer $(\mathrm{CP})$ containing $\mathrm{Cu}$ (II) has been prepared by self-assembly at the ambient temperature, and its chemical formula is $\left\{[\mathrm{Cu} \text { (BINDI) })_{0.5}\right.$ (bpe) $\left.] \cdot 3 \mathrm{H}_{2} \mathrm{O}\right\}_{n}(1)$. The as-prepared complex 1 has been fully determined via the single crystal X-ray diffraction, powder X-ray diffraction (PXRD), elemental analysis, Fourier transform infrared spectroscopy (FT-IR) and thermogravimetric analysis (TGA). The structural characterization results show that complex 1 shows a 2-fold interpenetrated framework with a 4,6-connected fsc-type topology. Afterward, the treatment effect of this compound against the Mycobacterium in alveolar macrophages was measured. The CFU assay results suggested that the compound could obviously decreased the Mycobacterium bacterial

\footnotetext{
*Correspondence to: Li-Li Diao, Division of Pulmonary Tuberculosis, the North Courtyard of Affiliated Hospital of Heibei University, Baoding, Heibei, CHINA

E-mail: lili_diao22@yeah.net

Accepted August 13, 2020 (received for review July 30, 2020)
}

Journal of Oleo Science ISSN 1345-8957 print / ISSN 1347-3352 online

http://www.jstage.jst.go.jp/browse/jos/ http://mc.manusriptcentral.com/jjocs 
numbers in alveolar macrophages. Besides, the expression level of rop $A$ gene in the alveolar macrophages was also inhibited through treated with the compound. The two types of atoms (nitrogen and oxygen) which are potentially to be able to interact with the protein have been studied in detail by employing molecular docking simulations.

\section{Experimental}

\subsection{Chemicals and measurements}

The materials utilized in this research were acquired from market and then they were utilized without additional purification unless otherwise specified. With the RINT2000 vertical goniometer, we can harvest the date of the powder $\mathrm{X}$-ray diffraction (PXRD) using the source of $\mathrm{Cu} \mathrm{Ka} \mathrm{X}$-ray (operated at $100 \mathrm{~mA}$ and $40 \mathrm{kV}$ ). We commonly can utilize the FT-IR spectrometer of Nicolet Impact 410 to record the FT-IR spectra from $4000 \mathrm{~cm}^{-1}$ to $400 \mathrm{~cm}^{-1}$ utilizing the $\mathrm{KBr}$ pellets. And through utilizing the Elemental analyzer of Perkin-Elmer 2400 to analyze the elements of Nitrogen, Hydrogen as well as Carbon. For the determinations of thermogravimetry (TGA), we can conduct that through utilizing the Mettler Toledo thermogravimetric analyzer with $40 \mathrm{~mL} \mathrm{~min}{ }^{-1}$ flow rate in atmosphere of $\mathrm{N}_{2}$ at $25-600^{\circ} \mathrm{C}$ temperature range (with the heating rate of $5^{\circ} \mathrm{C} \cdot \mathrm{min}^{-1}$ ). Record the whole fluorescence measurements by using the Hitachi F4500 fluorescence spectrophotometer.

\subsection{Preparation and characterization for $\left\{\left[\mathrm{Cu}(\mathrm{BINDI})_{0.5}\right.\right.$} (bpe) $] \cdot 3 \mathrm{H}_{2} \mathrm{O}{ }_{n}(1)$

Complex 1 was self-assembled at the ambient temperature utilizing the following process: stock the solutions of $0.2 \mathrm{mmol}$ and $0.048 \mathrm{~g} \mathrm{Cu}\left(\mathrm{NO}_{3}\right)_{2} \cdot 3 \mathrm{H}_{2} \mathrm{O}$ into the de-ionized water $(25 \mathrm{~mL}), 0.50 \mathrm{mmol}$ and $0.020 \mathrm{~g}$ of $\mathrm{NaOH}$ neutralized with $0.125 \mathrm{mmol}$ and $0.074 \mathrm{~g}$ of $\mathrm{H}_{4} \mathrm{BINDI}$ in the de-ionized water $(12.5 \mathrm{~mL})$ and $0.25 \mathrm{mmol}$ and $0.45 \mathrm{~g}$ bpe in the ethanol $(12.5 \mathrm{~mL})$ were synthesized. The solutions of $\mathrm{H}_{4} \mathrm{BINDI}$ and bpe were mixed and then stirred for five minutes. Afterward, this solution $(2 \mathrm{~mL})$ was layered carefully on metal solution $(2 \mathrm{~mL})$ utilizing $1.5 \mathrm{~mL}$ of the buffer solution (with the volume ratio of 1:2) of ethanol and deionized water. After 4 weeks, the blue crystals of the complex 1 with needle-shape were acquired and they were separated and cleaned thoroughly using the ethanol and deionized water. With $62 \%$ yield on the basis of $\mathrm{Cu}$ ions. FT-IR $\left(\mathrm{KBr}, 4000-600 \mathrm{~cm}^{-1}\right): 655(\mathrm{w}), 726(\mathrm{w}), 784(\mathrm{~m})$, $1025(\mathrm{w}), 1255(\mathrm{~m}), 1351(\mathrm{~s}), 1613(\mathrm{~s}), 1675(\mathrm{~m}), 1712(\mathrm{~m})$, 3451-3200 (bw). Elemental analysis for the complex 1: $\mathrm{C}_{27} \mathrm{H}_{15} \mathrm{~N}_{3} \mathrm{O}_{9} \mathrm{Cu}$ : calcd N, 7.13; H, 2.57; C, 55.06; found $\mathrm{N}$ : 6.94, H: 2.30, C: 54.98.

We can analyze the data of strength through software of crysalispro, after that transformed the intensity data into the files of HKL. We utilized diffractometer of Oxford Xcali-
burE to get the data of X-ray. Complex 1's original skeleton model was built through utilizing SHELXS based on the direct manner, and then modified the model through to SHELXL-2014 according to least square manner. Anisotropic parameters were mixed with complex 1 's atoms of nonhydrogen. Afterward, the entire hydrogen atoms are geometrically fixed to $\mathrm{C}$ atom they are linked to through utilizing the command of AFIX. Crystal Data for 1 $\left(\mathrm{C}_{27} \mathrm{H}_{20.5} \mathrm{CuN}_{3} \mathrm{O}_{8.75}\right)$ : monoclinic, space group P2/c (no. 13), $a=9.932(2) \AA, b=13.4120(10) \AA, c=19.9350(10) \AA, \beta=$ $97.326(2), V=2633.8(6) \AA^{3}, Z=4, T=293(2) \mathrm{K}, \mu(\mathrm{MoK} \alpha)$ $=0.888 \mathrm{~mm}^{-1}$, Dcalc $=1.489 \mathrm{~g} / \mathrm{cm}^{3}, 113172$ reflections measured $\left(5.118^{\circ} \leq 2 \Theta \leq 57.7^{\circ}\right), 6875$ unique $\left(R_{\text {int }}=0.0652\right.$, $\left.R_{\text {sigma }}=0.0254\right)$ which were used in all calculations. The final $R_{1}$ was $0.0568\left(\mathrm{I}>2 \sigma(\mathrm{I})\right.$ ) and $w R_{2}$ was 0.1672 (all data).

\subsection{Bacterial numbers counting}

After synthesizing the compound with novel structure, the treatment effect of compound against Mycobacterium tuberculosis induced via Mycobacterium was assessed with the CFU assay. This conducted was carried out under the instruction's guidance of with slight modifications. In brief, $50 \mathrm{BALB} / \mathrm{c}$ mice were obtained from the Jinan university (Jinan), and all the conduction in this present research were authorized via the Ethics Committee of the Jinan university (Jinan). All the animals were fed with free water and food at the standard condition before the experiment. Then, the mice were infected with $0.2 \mathrm{~mL}$ Mycobacterium $\left(10^{8} / \mathrm{mL}\right)$. After that the compound was given to mice for the treatment, with the Rifampin utilized as a positive control drug. Finally, the number of bacteria in alveolar macrophages was counted through the plate approach. This experiment needed to implement for three times or more.

\section{4 ropA gene expression}

The real time RT-PCR method was conducted in this current experiment for the expression determination of ropA gene. This conduction was accomplished on the basis of the protocols with slight modifications. In brief, the mice were infected with $0.2 \mathrm{~mL}$ Mycobacterium $\left(10^{8} / \mathrm{mL}\right)$. After that the compound was given to mice for the treatment, with the Rifampin utilized as a positive control drug. Next, the Mycobacterium in alveolar macrophages were separated, and we extracted the overall RNA in bacteria with the Trizol reagent. According to the recommendation of manufacturer, RNA was quantified and then it was transcribed reversely into cDNA using the kit. Then the SYBR Green Master Mix (Roche) was utilized for the preformation of RT-PCR, and the relative ropA gene expression level the in Mycobacterium was detected, and the $16 \mathrm{~s}$ gene was used as the internal control. The $2^{-\Delta \mathrm{CCt}}$ method was used to analyze the results for three times. 


\subsection{Docking methods}

The Lamarckian genetic algorithm (Lamarckian GA) has been utilized to scoring the binding conformations of the given ligand and the protein. The ligand in the current study is the Se metal complex which contains nitrogen and oxygen atoms as the potential active sites, the 4NOI (protein data bank ID) from the RNA polymerase subunit alpha family has been chosen to be the probe protein. The structure of the complex is obtained from the experimental results and the probe protein is got from the protein data bank. Both of the structures are used without additional optimization. A cuboid grid box has been used to cover the active sites of the 4NOI structure, the dimension of the grid box is $70,70,100$, and the coordinates of the grid center is $11.007,11.361,14.759$, all units in the molecular docking simulation is angstrom if not mentioned specifically. All molecular docking simulation has been performed by AutoDock4 software, the visualization has been done by PyMOL.

\section{Results and Discussion}

\subsection{Molecular structures}

In accordance with the data of crystal which harvested at ambient temperature, the structural solution as well as refinement results indicated that complex 1 is crystallized in monoclinic with space group of $\mathrm{P} 2 / c$, and it reveals a three-dimensional 2 -fold interpenetrated network com- posed of the bpe spacer and a $[\mathrm{BINDI}]^{4-}$ anion. Its asymmetric unit contains one bpe spacer, one $\mathrm{Cu}$ (II) ion, and 0.5 ligand of BINDI as well as four crystal guest molecules of water(Fig. 1a). The geometry around the center of $\mathrm{Cu} 1$ shows a distorted octahedron, which composed of four carboxylic acid(i.e., O4, O3, $\mathrm{O} 2$ and O1) oxygens in three distinct ligands of BINDI and two nitrogen atoms (i.e., N2 and N1) come from a bpe spacer leading to a 14-linked uninodal network. The $\mathrm{Cu}(\mathrm{II})$ ions are held together via the BINDI ligands along the bc plane to afford the 2D layered structure with large square windows (Fig 1b). These two-dimensional layers are in-depth extended via the ligands of bpe to generate a three-dimensional skeleton with $12.4 \times 5.5 \AA^{2}$ large one-dimensional channels (Fig. 1c). The existence of this large one-dimensional channel promotes the penetration of another three-dimensional network into the gap, thus forming a 2-fold interpenetrated three-dimensional skeleton (Fig. 1d). The analysis of TOPOS proves the existence of $\left\{3^{33} \cdot 4^{57} \cdot 6\right\}$ as the vertex symbol of interpenetration. Fascinatingly, owing to this interpenetration, the pore size is significantly decreased to $4.6 \times 2.5 \AA^{2}$. In addition, after the elimination of guest water molecules, the solventaccessible void of each unit detected via the PLATON was decreased to $11.9 \%\left(326.8 \AA^{3}\right)$. This structure also possesses the interaction of $\mathrm{C}-\mathrm{H} \cdots \mathrm{O}$ hydrogen bond, including the interaction between aromatic $\mathrm{C}-\mathrm{H}$ bonds from bpe connector and carboxylic acid oxygens(i.e., O4 and O3)in the ligand of BINDI, and oxygen atom $(\mathrm{O} 3 \mathrm{w})$ from the guest molecule of water.
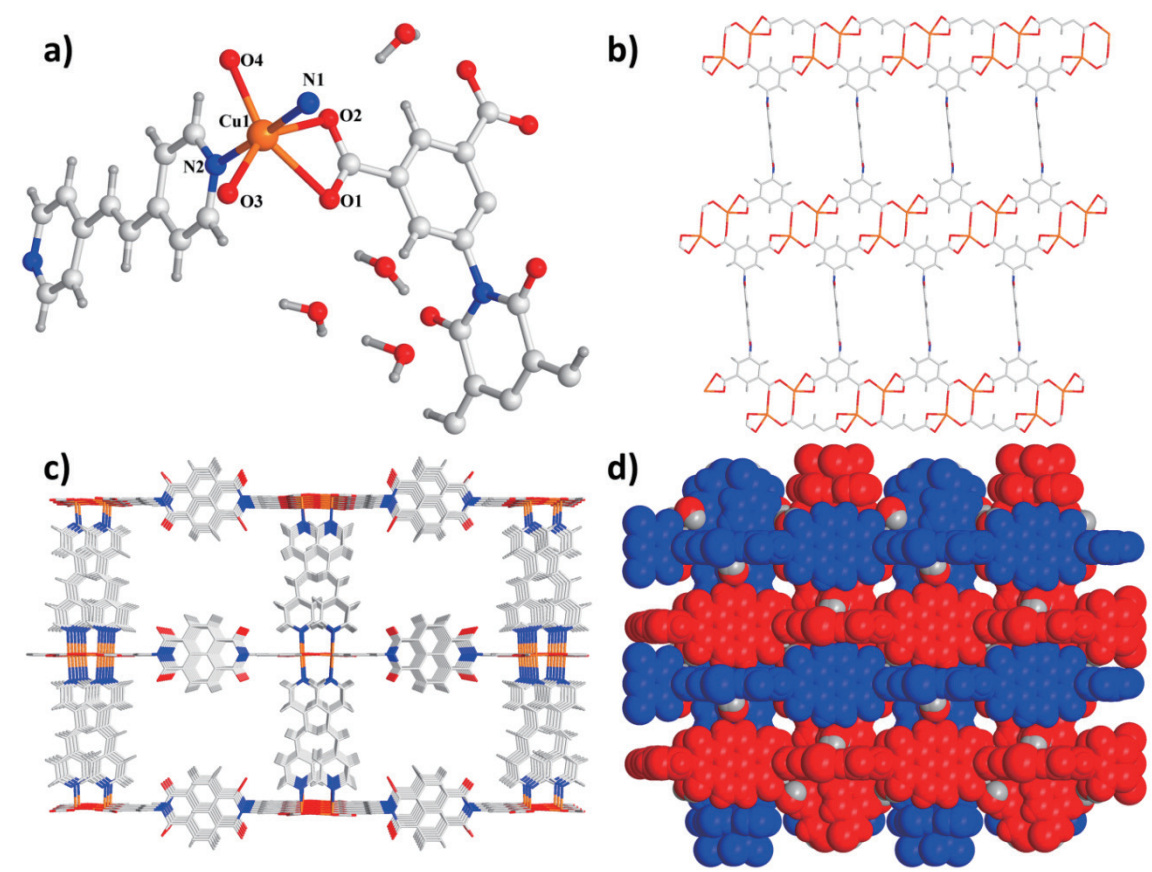

Fig. 1 (a) The complex 1's asymmetry unit view. (b) The complex 1's two-dimensional layered network along the plane bc. (c) The complex 1's single network displaying the large one-dimensional channels. (d) The three-dimensional 2-fold interpenetrated networks displaying the small one-dimensional channels. 


\subsection{PXRD measurements and TGA profiles}

For the sake of ascertaining complex 1's crystalline phase purity, PXRD experiments were performed on complex 1. It can be seen from the PXRD patterns (Fig. 2a) that the PXRD pattern of the complex 1 is consistent with the simulated diagram for the single crystal data, demonstrating there is a main crystal phase lie in relevant coordination polymer. The strength difference may be because of the crystal samples preferred orientation, which has been well documented in the previous literatures ${ }^{17,18)}$. Considering the following bioactivity tests, it is necessary to study the framework integrity in the simulated experimental conditions. To achieve this target, $100 \mathrm{mg}$ of the freshly as-prepared samples were grounded and then dispersed in the phosphate buffer saline (PBS) solution thoroughly via the ultrasonic treatment. After one day, the powder of 1 was separated via centrifugation and then the corresponding PXRD patterns were collected. As shown in the Fig. 2a, a good match between the PXRD patterns of the treated samples and those of the as-prepared ones could be found, indicating its good framework robustness in the simulated experimental conditions. Thermogravimetric analysis (TGA) of the as-prepared complex 1 was conducted on the thermogravimetric analyzer of Mettler Toledo under an atmosphere of $\mathrm{N}_{2}$ (with $40 \mathrm{~mL} \mathrm{~min}^{-1}$ flow rate) at $25^{\circ} \mathrm{C}-600^{\circ} \mathrm{C}$ temperature range (with $5^{\circ} \mathrm{C} \cdot \mathrm{min}^{-1}$ heating rate, Fig. 2b). The complex 1's TGA curve indicates a $5.8 \%$ weightlessness between $25^{\circ} \mathrm{C}$ and $150^{\circ} \mathrm{C}$ owing to the loss of three guest molecules of water. Moreover, a second $50.3 \%$ weightlessness (with the calculated value of $50.27 \mathrm{wt} \%$ ) at $220-515^{\circ} \mathrm{C}$ temperature range equivalents to the loss of the ligand of BINDI, and an indepth temperature increase resulted in the decomposition of skeleton.

\subsection{Compound could reduce the CFU numbers of the Mycobacterium in alveolar macrophages}

After the compound synthesis, the protective activity of the compound on the Mycobacterium tuberculosis was evaluated firstly. The dilution coated plate method was finished in this present study. According to the results illus-
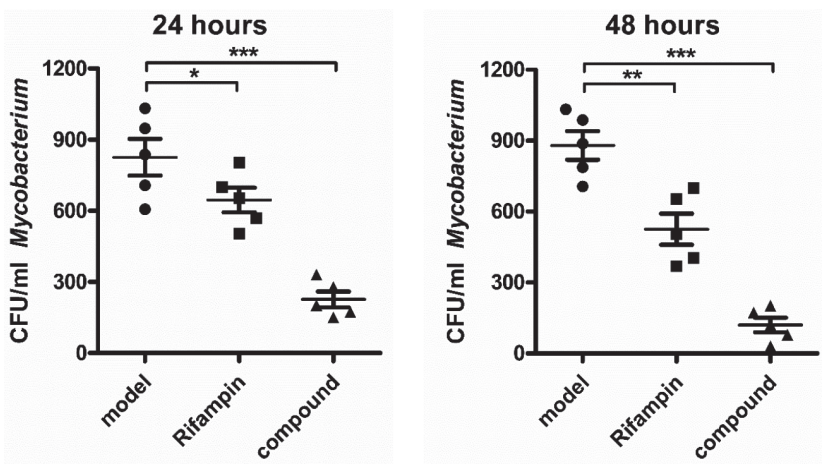

Fig. 3 Reduce CFU numbers of the Mycobacterium in alveolar macrophages after treated via the compound. Mice were infected with $0.2 \mathrm{~mL}$ Mycobacterium $\left(10^{8} / \mathrm{mL}\right)$, and the compound was given to mice for the treatment, with the Rifampin utilized as a positive control drug. The number of bacteria in alveolar macrophages was counted through using the plate approach.

trated in the Fig. 3, in the mice infected with Mycobacterium, there was an obviously increased the Mycobacterium numbers level in alveolar macrophages. However, after compound treatment, the bacterial numbers were significantly reduced, which is even better than the positive drug Rifampin.

\subsection{Compound inhibited the expression levels of ropA gene in Mycobacterium}

From the above results, we can see that compound possesses outstanding inhibitory effect against the Mycobacterium tuberculosis by reducing the Mycobacterium survival, which is even better than the positive drug Rifampin. So, in this research, we aimed to explore the related mechanism. According to the results illustrated in the Fig. 4, we can find the rop $A$ gene, an important gene for the bacterial survival was significantly reduced by the compound treatment. Compared with our former research, the inhibition of compound is stronger that the Rifampin. a)

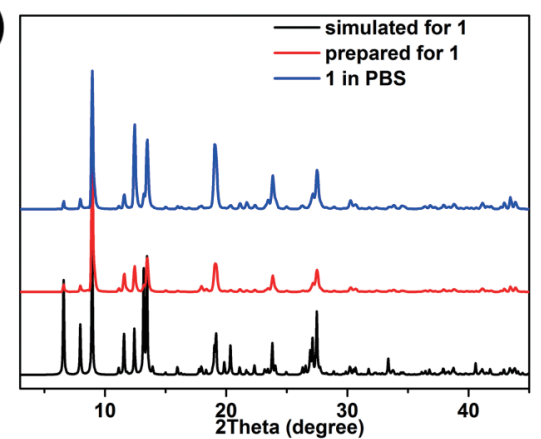

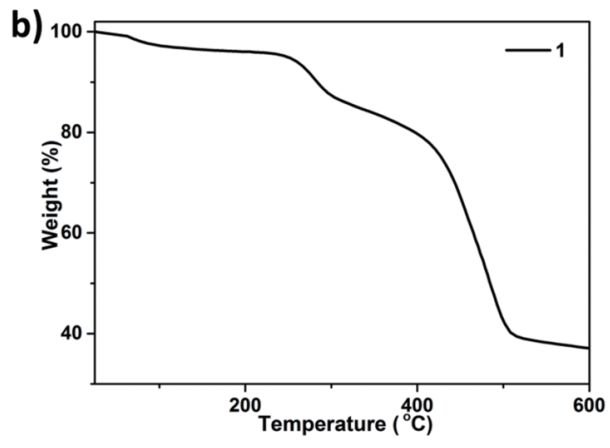

Fig. 2 (a) The complex 1's pattern of PXRD. (b) The curve of TGA for the complex 1. 

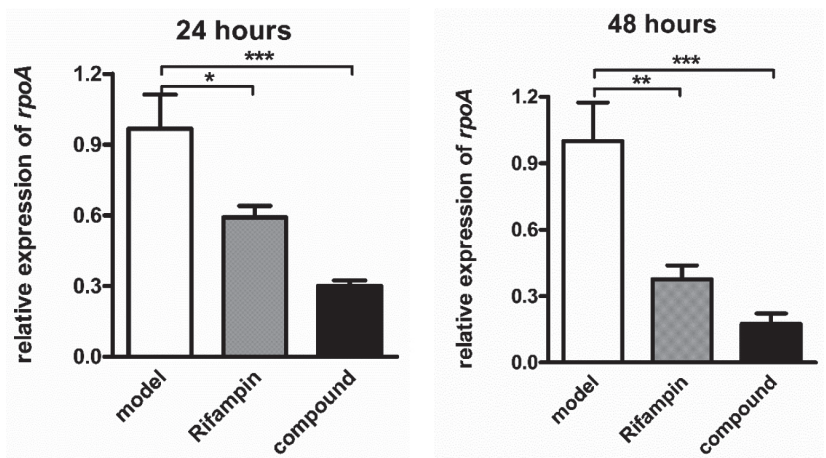

Fig. 4 Inhibited expression levels of ropA gene in Mycobacterium after treated via the compound. Mice were infected with $0.2 \mathrm{~mL}$ Mycobacterium $\left(10^{8} / \mathrm{mL}\right)$, and the compound was given to mice for the treatment, with the Rifampin utilized as a positive control drug. The relative $\operatorname{rop} A$ gene expression in the Mycobacterium was determined through the RT-PCR.

\subsection{Molecular docking}

As has been mentioned in the previous sections, there are two types of atoms which have the potential to be able to form binding interactions towards to the probe protein, the nitrogen and the oxygen atoms. In order to look into the details of the binding mechanism of the metal complex, molecular docking simulation has been performed to estimate the binding conformations. The binding conformation which has the lowest binding energy are analyzed further from the molecular resolution. However, in the current study, we found that there are two conformations which have very similar binding energies $(-7.56$ and $-7.55 \mathrm{kcal} /$ $\mathrm{mol}$ ), and have been displayed in Fig. 5. From both conformations we can see that only oxygen atoms are forming binding interactions with the probe protein, interestingly, the nitrogen atoms have no effect on the probe protein. Such results can be explained by the fact that the nitrogen atoms are mostly anticipated into the binding interaction of the metal center. Residue GLY-150, ARG-45, TYR-35 and THR-174 from the first conformation(Fig. 5a) have binding interactions with the metal complex, and moderate strength of binding interactions are observed, the binding distances varies from 2.2 to 3.3 (as has been mentioned in the simulation details section, the length unit in the simulation part is angstrom). In Fig. 5b, residues ARG-44, HIS-41 and TYR-181 are found to be interacting with the metal complex, the binding strength is also observed as moderate. The molecular docking results indicate that the metal complex is active for biological activity, and the origin of such activity is from the oxygen atoms on the complex.

\section{Conclusion}

In conclusion, we have triumphantly synthesized a novel coordination polymer containing $\mathrm{Cu}$ (II) by room temperature self-assembly approach and structurally characterized it through the analysis of element, powder X-ray diffraction, and single crystal X-ray diffraction, thermogravimetric analysis (TGA) as well as Fourier transform infrared spectra (FT-IR). The structural determination studies show that complex 1 shows a 2-fold interpenetrated three-dimensional skeleton structures with one-dimensional $4.6 \times 2.5 \AA^{2}$ channels. The CFU assay results suggested that compound could obviously decreased the Mycobacterium bacterial numbers in alveolar macrophages. Besides, the expression level of ropA gene in the alveolar macrophages was also inhibited by the compound treatment. Finally, we got this conclusion, the compound revealed outstanding treatment effect against Mycobacterium tuberculosis by inhibiting the expression level of ropA gene in Mycobacterium and reducing the bacterial survival rate in the macrophages. Molecular docking results found that the metal complex is active for biological activity, and the origin of such activity

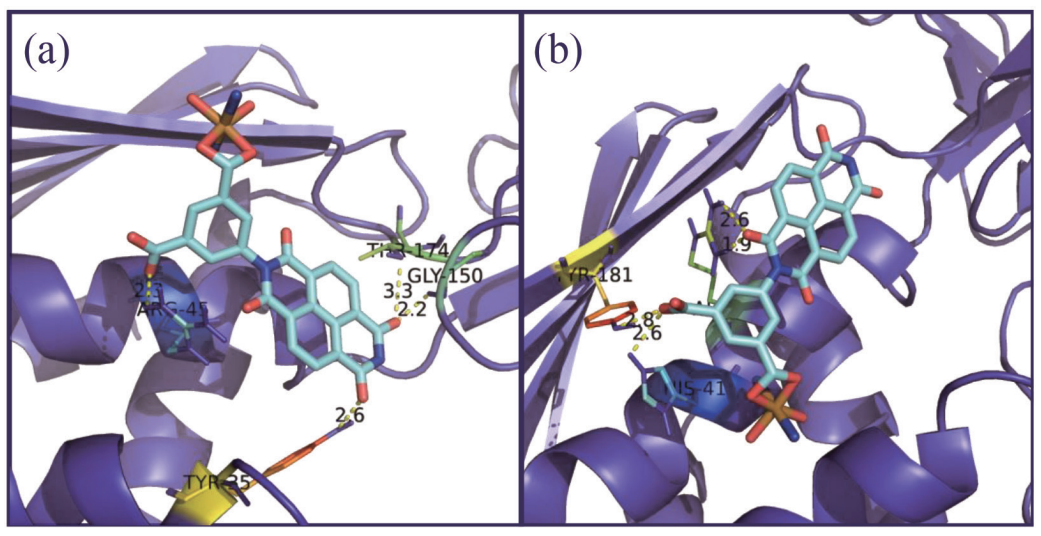

Fig. 5 The two binding conformations which have very similar binding energies are displayed, the binding energies are -7.56 (a) and -7.55 (b) kcal/mol. The Se metal complex is plotted in stick model, the interacting residues from the probe protein are plotted in line model and the probe protein itself is plotted as cartoon model. 
is from the oxygen atoms on the complex.

\section{Conflicts of Interest}

The author $(\mathrm{s})$ declare (s) that there is no conflict of interest regarding the publication of this paper.

\section{Data Availability}

The data used to support the findings of this study are included within the article.

\section{Acknowledgments}

Not applicable.

\section{Reference}

1) Menardo, F.; Duchêne, S.; Brites, D.; Gagneux, S. The molecular clock of Mycobacterium tuberculosis. PLoS Pathog. 15, e1008067(2019).

2) Nardell, E.A. Preventing transmission of Mycobacterium tuberculosis-A refocused approach. Clin. Chest. Med. 40, 857-869 (2019).

3) Fellag, M.; Loukil, A.; Saad, J.; Lepidi, H.; Bouzid, F.; Brégeon, F.; Drancourt, M. Translocation of Mycobacterium tuberculosis after experimental ingestion. PLoS One 14, e0227005 (2019).

4) Feng, X.; Feng, Y.Q.; Guo, N.; Sun, Y.L.; Zhang, T.; Ma, L.F.; Wang, L.Y. Series d-f heteronuclear metal-organic frameworks: Color tunability and luminescent probe with switchable properties. Inorg. Chem. 56, 17131721 (2017).

5) Feng, X.; Li, R.F.; Wang, L.Y.; Ng, S.W.; Qin, G.Z.; Ma, L.F. A series of homonuclear lanthanide coordination polymers based on a fluorescent conjugated ligand: syntheses, luminescence and sensor. CrystEngComm 17, 7878-7887 (2015).

6) Feng, X.; Feng, Y.Q.; Liu, L.; Wang, L.Y.; Song, H.L.; $\mathrm{Ng}, \mathrm{S} . \mathrm{W}$. A series of Zn-4f heterometallic coordination polymers and a zinc complex containing a flexible mixed donor dicarboxylate ligand. Dalton T. 42, 77417754 (2013).

7) Zhang, D.; Wang, M.M.; Jiang, N.; Liu, Y.; Yu, X.N.; Zhang, H.B. Electrochemical corrosion behavior of Nidoped ZnO thin film coated on low carbon steel substrate in $3.5 \% \mathrm{NaCl}$ solution. Int. J. Electrochem. Sci. 15, 4117-4126 (2020).
8) Arshad, M.; Du, H.L.; Javed, M.S.; Maqsood, A.; Ashraf, I.; Hussain, S.; Ma, W.L.; Ran, H.P. Fabrication, structure, and frequency-dependent electrical and dielectric properties of Sr-doped $\mathrm{BaTiO}_{3}$ ceramics. Ceram. Int. 46, 2238-2246 (2020).

9) Guo, M.Y.; Zhang, X.; Zhao, L.; Li, Y.K.; Chen, D.Y.; Yang, G.W.; Li, Q.Y. Regulation of deprotonation of 3,3di (1H-tetrazol-5-yl) pentanedioic acid: Solvothermal synthesis of $\mathrm{La}$ (III) and heterometallic $\mathrm{La}(\mathrm{III}) / \mathrm{Cu}$ (II) compounds for ablation of A549 cells. J. Solid State Chem. 259, 104-109 (2018).

10) Rohini, G.; Haribabu, J.; Aneesrahman, K.N.; Bhuvanesh, N.S.P.; Ramaiah, K.; Karvembu, R.; Sreekanth, A. Half-sandwich $\mathrm{Ru}$ (II) ( $\eta$-p-cymene) complexes bearing $N$-dibenzosuberenyl appended thiourea for catalytic transfer hydrogenation and in vitro anticancer activity. Polyhedron 152, 147-154 (2018).

11) Chen, D.M.; Zhang, X.J. A polyhedron-based metal-organic framework with a rare hexanuclear $\mathrm{Co}$ (II) cluster for selective sorption and chemical conversion for $\mathrm{CO}_{2}$. J. Solid State Chem. 278, 120906 (2019).

12) Yang, Y.; Xu, L.; Zhu, W.; Feng, L.; Liu, J.; Chen, Q.; Dong, Z.; Zhao, J.; Liu, Z.; Chen, M. One-pot synthesis of pH-responsive charge-switchable PEGylated nanoscale coordination polymers for improved cancer therapy. Biomaterials 156, 121-133(2018).

13) Senthil Raja, D.; Ramachandran, E.; Bhuvanesh, N.S.P.; Natarajan, K. Synthesis, structure and in vitro pharmacological evaluation of a novel 2-oxo-1,2-dihydroquinoline-3-carbaldehyde ( 2 '-methylbenzoyl $)$ hydrazone bridged copper(II) coordination polymer. Eur. J. Med. Chem. 64, 148-159(2013).

14) Munteanu, C.R.; Suntharalingam, K. Advances in cobalt complexes as anticancer agents. Dalton T. 44, 13796-13808(2015).

15) Raja, D.S.; Bhuvanesh, N.S.P.; Natarajan, K. A novel water soluble ligand bridged cobalt(ii) coordination polymer of 2-oxo-1,2-dihydroquinoline-3-carbaldehyde (isonicotinic) hydrazone: Evaluation of the DNA binding, protein interaction, radical scavenging and anticancer activity. Dalton T. 41, 4365-4377 (2012).

16) Jagadeesan, S.; Balasubramanian, V.; Baumann, P.; Neuburger, M.; Häussinger, D.; Palivan, C.G. Water-soluble Co (III) complexes of substituted phenanthrolines with cell selective anticancer activity. Inorg. Chem. 52, 12535-12544 (2013).

17) Pan, H.; Sun, H.; Poh, C.; Feng, Y.; Lin, J. Single-crystal growth of metallic nanowires with preferred orientation. Nanotechnology 16, 1559-1564(2005).

18) Chen, D.M.; Zhang, X.J. Stepwise and hysteretic sorption of $\mathrm{CO}_{2}$ in polycatenated metal-organic frameworks. CrystEngComm 21, 4696-4700 (2019). 